\title{
ON THE COEFFICIENTS OF STARLIKE FUNCTIONS
}

\author{
FINBARR HOLLAND
}

ABSTRACT. Every probability measure $\mu$ on the circle group generates a function $f$ that is starlike univalent on the open unit disc $\Delta$. In this note the relatiorship between $\left(c_{n}\right)$, the FourierStieltjes coefficients of $\mu$, and $\left(a_{n}\right)$, the Taylor coefficients of $f$, is examired. A number of theroems are presented which indicate (possibly in the presence of fairly mild restrictions) that the sequences $\left(c_{n}\right)$ and $\left(n a_{n}\right)$ behave similarly. For example, it is shown that if $f(\Delta)$ is finite, then $\left(c_{n}\right)$ converges to zero if, and only if, $\left(n a_{n}\right)$ converges to zero, thereby completing a result of Pommerenke.

1. Introduction and results. Let

$$
f(z)=z+\sum_{n=2}^{\infty} a(n) z^{n}
$$

be starlike on the open unit disc $\Delta$. Then, as is well known, there is a uniquely determined probability measure $\mu$ defined on the Borel subsers of the unit circle $\Gamma$ such that

$$
F(z)=\frac{z f^{\prime}(z)}{f(z)}=\int \frac{1+z \bar{\gamma}}{1-z \bar{\gamma}} d \mu(\gamma) \quad(z \in \Delta) .
$$

Defining the sequence $(c(n))$ by

$$
c(n)=2 \int \bar{\gamma}^{n} d \mu(\gamma) \quad(n=0,1,2, \cdots),
$$

it follows quickly from (2) that

$$
f(z)=z \exp \left(\sum_{n=1}^{\infty} c(n) z^{n} / n\right),
$$

and that the sequences $(a(n))$ and $(c(n))$ are linked by means of the following equations:

$$
(n+1) a(n)=\sum_{k=1}^{n_{n}} a(k) c(n-k) \quad(n=1,2, \cdots),
$$

where, for convenience, $a(1)=1$.

Received by the editors December 27, 1969 and, in revised form, May 10, 1971. AMS 1970 subject classifications. Primary 30A32; Secondary 30A18, 40E05.

Key words and phrases. Starlike function, univalent function, domain of finite area, probability measure, Banach space, closed linear span.

(c) American Mathematical Society 1972 
This convolution relationship suggests, among other things, that (possibly in the presence of additional requirements) the sequence $(n a(n))$ mimics the behaviour of $(c(n))$, and vice versa. One of the main aims of the present paper is to furnish evidence in support of this observation.

To begin with, if, for $1 \leqq p<\infty$, we denote by $l_{p}$ the Banach space of sequences $x=(x(n))$ with $\|x\|_{p}=\left(\sum_{n=0}^{\infty}|x(n)|^{p}\right)^{1 / p}$ as norm, we have:

THEOREM 1. Let $1 \leqq p<\infty$. Then the following statements are equivalent.

The sequence $(c(n))$ belongs to $l_{p}$.

The sequence $(n a(n))$ belongs to $l_{p}$.

Next, it follows trivially from (3) that $(c(n)) \in l_{\infty}$. It is shown in [2] that if the area of $f(\Delta)$ is finite, then also $(n a(n)) \in l_{\infty}$. If, in addition, $\mu$ is absolutely continuous (relative to normalised Lebesgue measure $m$ ), then, as Pommerenke has shown in [5], $\lim n a(n)=0$. We complete Pommerenke's analysis by establishing the following more precise statement.

THEOREM 2. Let $f$ map $\Delta$ onto a domain of finite area. Then

$$
\lim n a(n)=0
$$

if, and only if,

$$
\lim c(n)=0 .
$$

Because of the way in which $f$ and $\mu$ are related, it might be thought that condition (9), together with the assumption that the area of $f(\Delta)$ is finite, forces $\mu$ to be absolutely continuous; but this is not so. Indeed, there is a continuous probability measure $\mu$ that is singular with respect to $m$, and such that $c(n)=O\left(n^{-1 / 2+\varepsilon}\right)$ for every $\varepsilon>0$ (cf. Theorem 10.12 of [8]). It follows that the function $f$ defined by (4) is starlike and bounded. Thus, for this function, the hypothesis of the theorem is realised and $\lim c(n)=0$; but $\mu$ is singular.

Because of its independent interest, we have isolated the essential part of Theorem 2 as a separate result. Befeore stating this, we recall that the radial limit function $\hat{f}$ defined on $\Gamma$ by $\hat{f}(\gamma)=\lim _{r \rightarrow 1} f(r \gamma)(\gamma \in \Gamma)$ exists everywhere on $\Gamma$, though it can be infinite, but only on a set of Lebesgue measure zero.

THEOREM 3. With the hypothesis of the previous theorem, $\hat{f}$ belongs to $L^{2}(\mu)$ and the closed linear span of $\left\{\gamma^{n} \hat{f}(\gamma): n=0,1,2, \cdots\right\}$ coincides with the closed subspace spanned by $\left\{\gamma^{n}: n=1,2, \cdots\right\}$.

To show that (7) and (8) imply (6) and (9) respectively, we need to introduce the function

$$
g(z)=z / f(z) \quad(z \in \Delta) .
$$


Since $f$ is a normalised univalent function, $g$ is regular, bounded and nonzero on $\Delta$ [3]. The radial limits $\hat{g}(\gamma)=\lim _{r \rightarrow 1} g(r \gamma)(\gamma \in \Gamma)$ exist everywhere on $\Gamma$ and $\hat{g}(\gamma)=\gamma \mid \hat{f}(\gamma)$.

Also, the function $z \rightarrow z g(1 / z)$ maps $\{z:|z|>1\}$ onto a domain whose compact compiement $K$ is starlike. Thus, by classical results,

$$
\begin{gathered}
\text { area } K=\pi\left(1-\sum_{n=1}^{\infty} n|b(n)|^{2}\right) \geqq 0, \\
\text { where } g(z)=1+\sum_{n=0}^{\infty} b(n) z^{n+1} \quad(z \in \Delta) .
\end{gathered}
$$

Some further interesting properties of $g$ are summarised in the next few theorems.

THEOREM 4. In the above notation, area $K=\pi \int|\hat{g}|^{2} d \mu$.

THEOREM 5. Suppose that area $K=0$. Then

(13) the area of $f(\Delta)$ is infinite, and

(14) $\mu$ is singular with respect to $m$.

Conversely, if $\mu$ is discrete, then area $K=0$.

Examples show that neither (13) nor (14) imply that area $K=0$. On the one hand, if $f(z)=z /(1-z)$, then (13) holds; but area $K=\pi$. On the other hand, as we remarked above, there are continuous singular measures that induce bounded starlike functions. We are unable to decide whether or not (13) and (14) together force area $K=0$.

THEOREM 6. For $n=1,2,3, \cdots$,

$$
(n+1)|b(n)| \leqq 2 .
$$

Moreover, the inequality is strict for every $n$ unless either

$$
g(z)=1+b(0) z-\zeta^{-2} z^{2}
$$

where $|\zeta|=1,|b(0)| \leqq 2$ and $\operatorname{Re}(b(0) \zeta)=0$ or

$$
g(z)=\left(1-(\bar{\zeta} z)^{n+1}\right)^{2 / n+1},
$$

where $|\zeta|=1$ and $n \geqq 2$.

This result is basically only a very slight extension of a celebrated theorem of Clunie [1] who, however, makes the nonessential assumption that $b(0)=$ 0 . Oddly enough, though. it will emerge that we cannot have $(n+1)|b(n)|=$ 2 for $n \geqq 2$ unless, in the first place, $b(0)=0$. The establishment of this fact will be one of the novel features of our proof. We remark in passing that Pommerenke [7] also obtained the inequality (15) for arbitrary $b(0)$, but 
gave no discussion of the case of equality. The methods of the paper also yield the next result.

THEOREM 7. If either (i) area $K=0$ or (ii) $\lim c(n)=0$, then (iii) $\lim n b(n)=0$. Conversely, if $f$ maps $\Delta$ onto a domain of finite area, then (iii) implies (ii).

We omit a proof of this theorem, which strengthens a result given in [6], and mention only that neither of (i) and (ii) implies the other. To see that (i) does not imply (ii), consider $f(z)=z /(1-z)^{2}$. Again, (ii) is satisfied whenever $\mu$ is absolutely continuous, but then, by Theorem 5 , area $K \neq 0$.

Throughout the paper, $\mu$ will be a probability measure on $\Gamma, f, F$ and $g$ the functions defined by (2) and (10), and $(a(n)),(c(n))$ and $(b(n))$ the Taylor coefficients of these functions, as given by (1), (3) and (12) respectively.

2. Three lemmas. It will be convenient to have the following lemmas to hand. We begin with the statement of a well-known one and refer to [4] for a proof.

LEMMA 1. Let $\sum_{n=1}^{\infty} n|d(n)|^{2}<\infty$. If

then, as $n \rightarrow \infty$,

$$
h(z)=\sum_{n=0}^{\infty} d(n) z^{n} \quad(z \in \Delta)
$$

$$
\max _{\gamma \in \Gamma}\left|\sum_{k=0}^{n} d(k) \gamma^{k}-h(\gamma-\gamma / n)\right| \rightarrow 0 .
$$

LeMma 2. Write $b(-1)=1$ and define $\left(t_{n}\right)$ on $\Gamma$ by

$$
t_{n}(\gamma)=\sum_{k=0}^{n} b(k-1) \gamma^{k} \quad(n=0,1, \cdots)
$$

Then $\left(t_{n}\right)$ converges to $\hat{g}$ in (the norm of) $L^{2}(\mu)$.

Proof. Define $\left(g_{n}\right)$ on $\Gamma$ by $g_{n}(\gamma)=g(\gamma-\gamma / n)(n=1,2, \cdots)$. The boundedness of $g$ ensures that $\left(g_{n}\right)$ converges boundedly to $\hat{g}$. In view of (11) and Lemma $1,\left(t_{n}-g_{n}\right)$ converges to zero uniformly on $\Gamma$, and the conclusion follows readily from these facts.

LEMMA 3. Let the area of $f(\Delta)$ be finite. Then $\hat{f}$ belongs to $L^{2}(\mu)$ and, if $\left(s_{n}\right)$ is defined on $\Gamma$ by

$$
s_{n}(\gamma)=\sum_{k=1}^{n} a(k) \gamma^{k} \quad(n=1,2, \cdots),
$$

then $\left(s_{n}\right)$ converges to $\hat{f}$ in $L^{2}(\mu)$. 
Proof. Fix $r, 0 \leqq r<1$. Poisson's integral formula used in conjunction with Schwarz's inequality ensures that

$$
\left|f\left(r^{2} \gamma\right)\right|^{2} \leqq \int \frac{\left(1-r^{2}\right)|f(r \hat{\lambda})|^{2}}{|1-r \gamma \bar{\lambda}|^{2}} d m(\lambda) \quad(\gamma \in \Gamma)
$$

Consequently,

$$
\begin{aligned}
\int\left|f\left(r^{2} \gamma\right)\right|^{2} d \mu(\gamma) & \leqq \int|f(r \lambda)|^{2} d m(\lambda) \int \frac{\left(1-r^{2}\right)}{|1-r \bar{\gamma} \lambda|^{2}} d \mu(\gamma) \\
& =\int|f(r \lambda)|^{2} \operatorname{Re} F(r \lambda) d m(\lambda) \\
& =\sum_{n=1}^{\infty} n|a(n)|^{2} r^{2 n},
\end{aligned}
$$

by Fubini's theorem. (2) and Parseval's theorem. Since, as $r$ tends to 1 , $|f(r y)|$ increases to $|\hat{f}(\gamma)|$ for every $\gamma \in \Gamma$, the first assertion of the theorem follows from the last displayed inequality and the hypothesis.

Next, defining $\left(f_{n}\right)$ on $\Gamma$ by $f_{n}(\gamma)=f(\gamma-\gamma / n)(n=1,2, \cdots)$ we see that (i) $\hat{f}$ is the pointwise limit of $\left(f_{n}\right)$, and (ii) $\left|f_{n}-\hat{f}\right| \leqq 2|\hat{f}|(n=1,2, \cdots)$. Hence, by Lebesgue's dominated convergence theorem, $f_{n} \rightarrow \hat{f}$ in $L^{2}(\mu)$. But, by Lemma $1,\left(s_{n}-f_{n}\right)$ converges to zero in the uniform norm; and $\mu$ is finite. Hence $s_{n} \rightarrow \hat{f}$ in $L^{2}(\mu)$, as we wished to show.

3. Proof of Theorem 1. Let (6) obtain. Then $(c(n) / n) \in l_{1}$, and thence, by (4), $(a(n)) \in l_{1}$. A familiar application of Hölder's inequality to (5) now gives

$$
\|((n+1) a(n))\|_{p} \leqq\|(a(n))\|_{1}\|(c(n))\|_{p}
$$

Thus, (7) is true.

Conversely, if (7) holds, then certainly $(a(n)) \in l_{1}$. Since $1 / g$ does not vanish on $\Delta \cup \Gamma$, a classical theorem of Wiener now enables us to assert that $(b(n)) \in l_{1}$. But $F=g f^{\prime}$, and so

$$
c(n)=\sum_{k=1}^{n+1} k a(k) b(n-k) \quad(n=1,2, \cdots) .
$$

Appiying Hölder's inequality to this, in the same way as before, we infer that (6) is satisfied. This ends the proof.

4. Proof of Theorem 3. At any rate, by Lemma $3, \hat{f} \in L^{2}(\mu)$. So, denote by $M$ and $N$ the $L^{2}(\mu)$-closures of the subspaces spanned by $\left\{\gamma^{n} \hat{f}(\gamma)\right.$ : $n=0,1, \cdots\}$ and $\left\{\gamma^{n}: n=1,2, \cdots\right\}$ respectively. Then $\gamma M \subset M$ and $\gamma N \subset N$. Accordingly, to finish the proof of the theorem, it suffices to show that $\hat{f} \in N$ and that $\gamma \in M$. That $\hat{f} \in N$ is also a consequence of Lemma 3. 
That $\gamma \in M$ follows from Lemma 2 and the fact that $\gamma=\hat{f}(\gamma) \hat{g}(\gamma)$ a.e. $d \mu$. This completes the proof.

5. Proof of Theorem 2. Starting with (5), and taking account of (3) and (18), we have, for $n=1,2, \cdots$,

$$
\begin{aligned}
(n+1) a(n) & =2 \int \bar{\gamma}^{n} s_{n}(\gamma) d \mu(\gamma) \\
& =2 \int \bar{\gamma}^{n} \hat{f}(\gamma) d \mu(\gamma)+2 \int \bar{\gamma}^{n}\left(s_{n}(\gamma)-\hat{f}(\gamma)\right) d \mu(\gamma) .
\end{aligned}
$$

It results immediately from this and Lemma 3 that

$$
\lim n a(n)=0 \Leftrightarrow \lim \int \bar{\gamma}^{n} f(\gamma) d \mu(\gamma)=0 .
$$

But, by Theorem 3,

$$
\lim \int \bar{\gamma}^{n} d \mu(\gamma)=0 \Leftrightarrow \lim \int \bar{\gamma}^{n} f(\gamma) d \mu(\gamma)=0 ;
$$

and we are done.

6. Proof of Theorem 4. It follows without difficulty from the definitions that if $0 \leqq r<1$, then

$$
\begin{aligned}
1-\sum_{n=1}^{\infty} n|b(n)|^{2} r^{2 n+2} & =\int|g(r \gamma)|^{2} \operatorname{Re} F(r \gamma) d m(\gamma) \\
& =\left(1-r^{2}\right) \int d \mu(\lambda) \int \frac{|g(r \gamma)|^{2}}{|1-r \gamma \bar{\lambda}|^{2}} d m(\gamma) \\
& =\left(1-r^{2}\right) \int d \mu(\gamma) \sum_{n=0}^{\infty}\left|t_{n}(\gamma)\right|^{2} r^{2 n} \\
& =\left(1-r^{2}\right) \sum_{n=0}^{\infty} r^{2 n} \int\left|t_{n}(\gamma)\right|^{2} d \mu(\gamma) .
\end{aligned}
$$

Comparing coefficients of powers of $r$, we deduce that

$$
\begin{aligned}
1 & =\int\left|t_{1}(\gamma)\right|^{2} d \mu(\gamma), \\
1-\sum_{k=1}^{n-1} k|b(k)|^{2} & =\int\left|t_{n}(\gamma)\right|^{2} d \mu(\gamma) \quad(n=2,3, \cdots) .
\end{aligned}
$$

In view of (11), a direct appeal to Lemma 2 now completes the proof. 
7. Proof of Theorem 5. Suppose (13) is false; so that $\sum_{n=1}^{\infty} n|a(n)|^{2}<\infty$. Now, if $0 \leqq r<1$, then

and so

$$
\begin{aligned}
r & =\int \bar{\gamma}(r \gamma) \operatorname{Re} F(r \gamma) d m(\gamma) \\
& =\int \bar{\gamma} f(r \gamma) g(r \gamma) \operatorname{Re} F(r \gamma) d m(\gamma),
\end{aligned}
$$

$$
\begin{aligned}
r^{2} & \leqq \int|f(r \gamma)|^{2} \operatorname{Re} F\left(r \gamma^{\prime}\right) d m(\gamma) \int|g(r \gamma)|^{2} \operatorname{Re} F(r \gamma) d m(\gamma) \\
& =\left(\sum_{n=1}^{\infty} n|a(n)|^{2} r^{2 n}\right)\left(1-\sum_{n=1}^{\infty} n|b(n)|^{2} r^{2 n+2}\right),
\end{aligned}
$$

whence it follows that area $K \geqq\left(\pi / \sum_{n=1}^{\infty} n|a(n)|^{2}\right)>0$, contradicting the assumption of the theorem.

As regards (14) we note, by Theorem 4 , that $\int|\hat{g}|^{2} d \mu=0$. Hence $\hat{g}$ vanishes on a Borel set $E$ with $\mu(E)=1$. But $g$ is regular, bounded and not identically zero; so that $m(E)=0$, by the F. and M. Riesz theorem. Thus, $\mu$ is singular relative to $m$.

Finally, if $\mu$ is discrete, it is concentrated on a countable subset $\Omega$ of $\Gamma$. If $\omega \in \Omega$ it follows easily from (2) that

$$
r \frac{d}{d r} \log |f(r \omega)|=\operatorname{Re} F(r \omega) \geqq \frac{1+r}{1-r} \mu(\{\omega\})
$$

and so $|f(r \omega)| \rightarrow \infty$ as $r \rightarrow 1$. Consequently, $\hat{g}$ vanishes on $\Omega$ and the conclusion follows.

8. Proof of Theorem 6. Since $z g^{\prime}(z)=F(z)(1-g(z))(z \in \Delta)$, the coefficients of $g$ are related to those of $F$ by the system of equations

$$
\begin{aligned}
b(0) & =-c(1), \\
(n+1) b(n) & =-c(n+1)-\sum_{k=0}^{n-1} b(k) c(n-k) \quad(n=1,2, \cdots) .
\end{aligned}
$$

Therefore,

$$
(n+1) b(n)=-2 \int \bar{\gamma}^{(n+1)} t_{n}(\gamma) d \mu(\gamma) \quad(n=1,2, \cdots) .
$$

Hence,

$$
(n+1)^{2}|b(n)|^{2} \leqq 4 \int\left|t_{n}\right|^{2} d \mu=4\left(1-\sum_{k=1}^{n-1} k|b(k)|^{2}\right) \leqq 4,
$$

by Schwarz's inequality and (19), whence (15) follows. 
Assume that $(n+1)|b(n)|=2$ for some $n \geqq 1$ and choose $\zeta \in \Gamma$ so that $(n+1) b(n)=-2 \bar{\zeta}^{(n+1)}$. We observe first from $(22)$ that $b(1)=\cdots=$ $b(n-1)=0$; so that $t_{k}=t_{1}$ if $1 \leqq k \leqq n$. Next, by $(21)$,

$$
\int \bar{\gamma}^{(n+1)} t_{1}(\gamma) d \mu(\gamma)=\xi^{(n+1)}
$$

But $\int\left|t_{1}\right|^{2} d \mu=1$, and so

$$
\zeta^{(n+1)} t_{1}(\gamma)=\gamma^{n+1} \text { a.e. } d \mu \text {. }
$$

If $n=1$, then, since $\mu$ is not the zero measure, $\gamma^{2}-\zeta^{2}(1+b(0) \gamma)$ has two zeros on $\Gamma$. Consequently, it follows that $i b(0) \zeta$ is real, that $|b(0)| \leqq 2$ and that $g$ is of the form (16), as stated.

Otherwise, $n \geqq 2$, in which case (23) used in combination with (21) implies that $c(k)=0$, if $1 \leqq k \leqq n$. Thus, by $(20), b(0)=0$. Returning to (23) and noting that now $t_{1}=1$, we infer that $\mu$ is concentrated on the set $\left\{\zeta, \zeta \omega, \cdots, \zeta \omega^{n}\right\}$, where $\omega^{n+1}=1, \omega \neq 1$. Since $c(k)=0$ if $1 \leqq k \leqq n, \mu$ has mass $1 /(n+1)$ at each point of this set, and a straightforward analysis of (2) shows that $g$ reduces to (17). The proof is complete.

I wish to thank the referee for his comments.

\section{REFERENCES}

1. J. Clunie, On meromorphic schlicht functions, J. London Math. Soc. 34 (1959), 215216. MR 21 \#5737.

2. J. Clunie and F. R. Keogh, On starlike and convex schlicht functions, J. London Math. Soc. 35 (1960), 229-233. MR 22 \#1682.

3. W. Hayman, Multivalent functions, Cambridge Tracts in Math. and Math. Phys., no. 48, Cambridge Univ. Press, Cambridge, 1958. MR 21 \#7302.

4. F. Holland and D. K. Thomas, The area theorem for starlike functions, J. London Math. Soc. (2) 1 (1969), 127-134. MR 39 \#7080.

5. Ch. Pommerenke, On starlike and convex functions, J. London Math. Soc. 37 (1962), 209-224. MR 25 \#1279.

6. - Über einige Klassen meromorpher schlichter Funktionen, Math. Z. 78 (1962), 263-284. MR 28 \#1285.

7. - On meromorphic starlike functions, Pacific J. Math. 13 (1963), 221-235. MR $27 \# 280$.

8. A. Zygmund, Trigonometrical series. Vol. 2, 2nd ed., Cambridge Univ. Press, New York, 1959. MR 21 \#6498.

Department of Mathematics, University College, Cork, Ireland 